\title{
Custeio Baseado em Atividade e Tempo (TDABC) Aplicado em Lavanderia: Estudo de Caso
}

Mariélly Warmeling Laucsen Martins
Mestrado em andamento pela Universidade Comunitária Regional de Chapecó -
UNOCHAPECÓ Avenida Senador Attílio Fontana, 591-E. Efapi. Chapecó/SC. CEP: 89.809-000 E-mail: mariellylaucsen@gmail.com

Clarice Flor Marçal Graduada em Administração de Empresas - UNISUL Avenida José Acácio Moreira, 787. Bairro Dehon. Tubarão/SC. CEP: 88.700-000 E-mail: clariceflor1989@gmail.com

Rodney Wernke Doutorado em Engenharia de Produção pela Universidade Federal de Santa Catarina UFSC Professor da Universidade do Sul de Santa Catarina - UNISUL Professor da Universidade Comunitária Regional de Chapecó - UNOCHAPECÓ Avenida Senador Attílio Fontana, 591-E. Efapi. Chapecó/SC. CEP: 89.809-000 E-mail: rodneywernke1@hotmail.com

Evandro Bonetti Mestrado em andamento pela Universidade Comunitária Regional de Chapecó UNOCHAPECÓ Avenida Senador Attílio Fontana, 591-E. Efapi. Chapecó/SC. CEP: 89.809-000 E-mail:ebonetti@gmail.com

Letícia Sandrin Especialização em andamento em Gestão Estratégica e Controladoria pela Universidade Comunitária Regional de Chapecó - UNOCHAPECÓ Avenida Senador Attílio Fontana, 591-E. Efapi. Chapecó/SC. CEP: 89.809-000

E-mail: leticia_sandrin@unochapeco.edu.br

\section{RESUMO}

O artigo pretende responder questão de pesquisa sobre como utilizar o Time-Driven Activity-Based Costing (TDABC) na determinação do custo da prestação de serviços de uma pequena lavanderia e tem o objetivo de calcular o custo dos serviços prestados por intermédio deste método de custeio. A metodologia utilizada na pesquisa pode ser caracterizada como descritiva, com abordagem qualitativa, por meio de um estudo de caso. Na revisão da literatura, foram abordados os conceitos inerentes, as etapas de implementação, as vantagens e as limitações associadas. $\mathrm{Na}$ apresentação e 
Custeio Baseado em Atividade e Tempo (TDABC) Aplicado em Lavanderia: Estudo de Caso

Mariélly Warmeling Laucsen Martins, Clarice Flor Marçal, Rodney Wernke, Evandro Bonetti, Letícia

Sandrin

discussão dos resultados, são relatados os passos seguidos para aplicar o TDABC na lavanderia, com descrição detalhada destes e das informações resultantes. Ainda evidencia-se como foi possível apurar o custo unitário de processar cada produto, conhecer o valor total de custo atribuído em razão do volume processado, além da identificação da ociosidade fabril mensal (em valor e em percentual do tempo disponível). Na parte final, são evidenciadas as conclusões oriundas, onde se constatou que o método é aplicável nessa entidade em sua configuração inicial, sem requerer adaptações significativas.

Palavras-chave: TDABC. Lavanderia. Estudo de caso.

Time-driven Activity-based Costing (TDABC) Applied in Laundry: Case Study

\section{ABSTRACT}

The article intends to answer a research question on how to use Time-Driven ActivityBased Costing (TDABC) in determining the cost of providing a small laundry service and has the objective of calculating the cost of services provided through this costing method. The methodology used in the research can be characterized as descriptive one, with a qualitative approach, through a case study. In the literature review the inherent concepts, the implementation stages, the advantages and the associated limitations were discussed. In the presentation and discussion of the results are reported the steps followed to apply the TDABC in the laundry, with detailed description of these and the resulting information. Also, it is evident how it was possible to determine the unit cost of processing each product, to know the total amount of cost attributed as result of the volume processed, as well as to identify the monthly factory idleness (in value and percentage of available time). In the final part, the conclusions are shown, where it was found that the method is applicable in this entity in its initial configuration, without requiring significant adaptations.

Keywords: TDABC. Laundry. Case study.

\section{INTRODUÇÃO}

Para o Instituto Brasileiro de Geografia e Estatística [IBGE] (2016), o setor de serviços é caracterizado por atividades bastante heterogêneas quanto ao porte das empresas, à remuneração média e à intensidade no uso de tecnologias. Nas últimas décadas, o desempenho das atividades que compõem o setor vem se destacando pelo dinamismo e pela crescente participação na produção econômica brasileira. No ano de 
Custeio Baseado em Atividade e Tempo (TDABC) Aplicado em Lavanderia: Estudo de Caso

Mariélly Warmeling Laucsen Martins, Clarice Flor Marçal, Rodney Wernke, Evandro Bonetti, Letícia

Sandrin

2013, segundo tal instituto, existiam no Brasil cerca de 1,2 milhão de empresas prestadoras de serviços, que geraram $R \$ 1,2$ trilhão em receita operacional líquida, empregavam 12,5 milhões de pessoas e pagaram aproximadamente $\mathrm{R} \$ 253,9$ bilhões de reais em salários.

Entretanto, outra pesquisa do IBGE (2012) evidenciou que a realidade empresarial brasileira apresenta uma dualidade: ao mesmo tempo que o número total de empresas ativas no país vem crescendo ano após ano, quase $50 \%$ das empresas que abrem as suas portas no Brasil terminam por encerrar suas atividades antes do terceiro ano de mercado.

Realidade assemelhada também se verifica especificamente em Santa Catarina, segundo Roldão (2013), visto que um estudo divulgado pelo Sebrae/SC (denominado Sobrevivência das Empresas) apontou que, a cada 100 companhias criadas no estado barriga-verde, em média apenas 76 sobrevivem aos dois primeiros anos de vida período considerado o mais crítico pela instituição em função das dificuldades de consolidar o negócio. Na região de Tubarão (onde foi realizado o estudo de caso deste artigo), esse índice foi de 77\%; em Criciúma, foi de 68\%; em Florianópolis, foi de 70\%; enquanto que a média nacional foi de $75,6 \%$. Provavelmente em virtude dessa realidade, estudos sobre os fatores que conduzem uma empresa a ter mais sucesso que outra no mercado tem sido uma preocupação constante nos âmbitos acadêmico, empresarial e governamental (Santos, Vasconcelos, Colares, \& Moreira, 2014).

Parte desse problema pode ser associado ao acirramento da concorrência, o que praticamente força que os gerentes das prestadoras de serviços priorizem a gestão de custos e preços de venda. Nesse sentido, Wernke, Junges e Luiz (2015) defendem que, para ter preços competitivos, é salutar que os custos de cada serviço sejam adequadamente mensurados para não prejudicar a lucratividade dos segmentos de mercado visados (serviços, linhas, tipos de clientes atendidos, entre outros) ou reduzir a competitividade perante os concorrentes.

Contudo, no caso de empresas que possuem processos complexos ou que empregam diversos equipamentos e funcionários a serem utilizados na prestação de serviços, a alocação dos custos indiretos às atividades executadas é mais complexa. 
Custeio Baseado em Atividade e Tempo (TDABC) Aplicado em Lavanderia: Estudo de Caso

Mariélly Warmeling Laucsen Martins, Clarice Flor Marçal, Rodney Wernke, Evandro Bonetti, Letícia

Sandrin

Para minimizar esse problema, podem ser utilizados métodos como Custeio por Absorção, Unidade de Esforço de Produção (UEP), Activity-Based Costing (ABC), Timedriven Activity-based Costing (TDABC) etc.

$\mathrm{Na}$ literatura referente ao tema, encontram-se citações acerca das vantagens e limitações associáveis a esses métodos, mas geralmente estão voltadas para aplicação em organizações fabris (ou de grande porte). Além disso, a escolha por uma dessas formas de custeamento depende de vários fatores relacionados à entidade que pretende adotá-los, especialmente em termos da adequação do método às características do(s) processo(s) requerido(s) pelo(s) serviço(s) prestado(s).

Especificamente no âmbito de lavanderias (foco deste estudo), o "tempo" de processamento nas máquinas costuma ser a variável principal a ser considerada, pois as etapas de produção necessárias para realizar determinados serviços podem demandar tempos distintos, conforme o tipo de item a ser processado (edredons, camisas, camisetas, ternos, tapetes etc.). Em decorrência disso, como o TDABC prioriza a variável "tempo", este provavelmente seria adequado para tal contexto. Nessa direção, corroboram a adequação desse método ao âmbito das prestadoras de serviços os estudos de Sarokolaei, Savizb, Moradlooc e Dahajd (2013), Werner, Bruggeman Sarens, Anderson e Levant (2008) e Dalmácio, Rezende e Aguiar (2007), por exemplo.

Porém, não foram encontradas publicações que discorressem sobre o uso do TDABC numa lavanderia, tipo de prestadora de serviços no qual um grande contingente de empresas é de pequeno porte, especialmente quando sediadas fora das capitais dos estados brasileiros. Por isso, uma pesquisa com tal ênfase pode justificar-se pelo ineditismo em termos do tipo de empresa abrangido, além de contribuir com o aprimoramento gerencial dessa modalidade de negócio comum nas cidades do país.

Por outro lado, o fato de nenhuma publicação anterior ter abordado a aplicação do TDABC em lavandeira pode ocasionar dúvidas sobre sua aderência a esse tipo de empreendimento ou sobre como aplicá-lo para apurar os custos dos serviços prestados nessas empresas. No sentido de dirimir tais incertezas, nesta pesquisa se pretende responder à seguinte pergunta: Como utilizar o TDABC na determinação do custo da prestação dos serviços de uma lavanderia de pequeno porte? Para tanto, fixou-se como 
Custeio Baseado em Atividade e Tempo (TDABC) Aplicado em Lavanderia: Estudo de Caso

Mariélly Warmeling Laucsen Martins, Clarice Flor Marçal, Rodney Wernke, Evandro Bonetti, Letícia

Sandrin

objetivo primordial do estudo calcular o custo dos serviços prestados por meio do método Time-Driven Activity-Based Costing.

O foco proposto pode ser justificado por dois ângulos. O primeiro está relacionado ao ponto de vista econômico, visto que as empresas de pequeno porte são grandes geradoras de empregos no Brasil, além de representarem parcela expressiva do PIB nacional.

O segundo ponto é que algumas pesquisas na área de custos, como Baldvinsdottir, Mitchell e Nørreklit (2010) e Lukka (2010), destacam que muitos estudos priorizam somente o aspecto teórico e que seria recomendável que um maior número de trabalhos acadêmicos tivesse como foco a efetiva ajuda prática para os gestores das empresas, principalmente aquelas classificáveis como de pequeno porte.

Acerca disso, Dorneles (2004) salienta que as pequenas empresas têm deficiências no controle dos seus custos em função de diversos fatores que as afetam (como, por exemplo, escassez de recursos e falta de controles formais ou contábeis). Por isso, boa parte das pequenas empresas define seus preços conforme a média do mercado, devido à competitividade, mas também em virtude da falta de conhecimento da composição de seus custos.

Essa realidade também está presente nas empresas que prestam serviços, conforme mencionado por Baxendale (2001), que registra o fato de muitas dessas organizações terem dificuldades para determinar a composição dos seus custos, especialmente porque grande parte desses gastos podem ser considerados como "custos fixos", o que dificulta a atribuição dos mesmos aos diversos serviços prestados pela entidade.

\section{REVISÃO DA LITERATURA}

As limitações atribuídas ao $A B C$ foram os principais motivadores do aparecimento do TDABC, conforme citado por Tseb e Gong (2009), Dalci, Tanis e Kosan (2010), Kont e Jantson (2011), Ratnatunga, Tseb e Balachandranc (2012) e Siguenza-Guzman (2014). 
Custeio Baseado em Atividade e Tempo (TDABC) Aplicado em Lavanderia: Estudo de Caso

Mariélly Warmeling Laucsen Martins, Clarice Flor Marçal, Rodney Wernke, Evandro Bonetti, Letícia

Sandrin

Destarte, é possível entender que Kaplan e Anderson (2004) desenvolveram o TDABC a partir da simplificação de determinados aspectos do $A B C$, bem como fizeram algumas inovações. Na primeira versão divulgada sobre o TDABC, seus idealizadores defenderam que essa forma de mensurar custos permitiria projetar a demanda de recursos gerada por transação, produto ou cliente, em vez de alocar os custos de recursos primeiro para atividades e, a seguir, para produtos ou clientes (como ocorre no $A B C$ ). Então, para cada grupo de recursos seria necessário determinar o custo por unidade de tempo (horas ou minutos) para suprir a capacidade de recursos e os tempos unitários de consumo da capacidade de recursos por produtos, serviços e clientes (os tempos de produção requeridos para cada produto). Referidos autores, então, argumentavam que isso ocasionaria o benefício de se obter taxas de direcionadores de custos mais confiáveis, visto que possibilitavam estimar tempos unitários até para transações complexas rapidamente (por meio das equações de tempo).

Mais adiante, Kaplan e Anderson (2007) mencionaram que o TDABC teria a capacidade de simplificar o trabalho de custeio por eliminar a necessidade de pesquisas e entrevistas com os funcionários para alocar os custos dos recursos às atividades, antes de direcioná-los aos objetos de custeio priorizados. Nesse caso, defendem que, no TDABC, os custos dos recursos são atribuídos de forma direta aos itens a custear, utilizando somente fatores de fácil estimativa: taxa do custo da capacidade e demanda de capacidade de recursos pelos objetos de custeio. Para tanto, são apurados os custos totais (em $\mathrm{R} \$$ ) a serem consumidos pelo departamento ou processo a ser custeado e que pode envolver folha de pagamentos, ocupação, equipamentos etc.

$\mathrm{Na}$ etapa seguinte, o valor desse custo total (em $\mathrm{R} \$$ ) é dividido pela capacidade prática dos departamentos ou processos (ou seja, ao tempo de trabalho disponível dos funcionários que efetivamente executam as atividades que consumiram esses recursos). Nesse caso, se calcula a taxa do custo de capacidade (em $\mathrm{R} \$$ ) dividindo o custo da capacidade disponibilizada (em $R \$$ ) pela capacidade prática fornecida (em horas ou minutos). Numa fase seguinte, a taxa do custo de capacidade (em $R \$$ ) é utilizada para distribuir os custos dos recursos departamentais entre os objetos a 
Custeio Baseado em Atividade e Tempo (TDABC) Aplicado em Lavanderia: Estudo de Caso

Mariélly Warmeling Laucsen Martins, Clarice Flor Marçal, Rodney Wernke, Evandro Bonetti, Letícia

Sandrin

custear e estimando a demanda da capacidade de recursos requerida pelos mesmos. Para essa finalidade, é utilizado o "tempo" de execução da atividade, motivo da denominação atribuída ao método. Dessa forma, o TDABC requer a estimativa dos tempos gastos para execução das atividades em cada processo e possibilita que 0 tempo estimado seja especificado levando em consideração as características das atividades abrangidas (maior ou menor complexidade, por exemplo).

Everaert e Bruggeman (2007) aduzem que, para implementar o TDABC, se deve: levantar os recursos fornecidos às atividades e separá-los em grupos (como folha de pagamentos, energia, depreciações, entre outros); apurar o valor gasto com cada recurso; determinar a capacidade prática das atividades; calcular o custo unitário de cada recurso ao dividir o valor encontrado para cada grupo de recursos pela capacidade prática da atividade; medir o tempo gasto para executar as atividades e multiplicar o custo unitário pelo tempo requerido para elaborar cada objeto de custo conforme as atividades requeridas.

Sarokolaei et al. (2013) relatam que a inovação trazida pelo TDABC está calcada basicamente em alocar os custos dos recursos diretamente aos objetos de custeio mediante estimativas de tempo. Para tanto, este exige a definição de apenas dois parâmetros: o custo por unidade de tempo e o volume de tempo consumido pelas atividades relacionadas aos objetos que se pretende custear.

A utilização das equações de tempo como base para atribuição de valor aos produtos ou serviços é fundamento básico do TDABC, conforme o entendimento de Barret (2005), Ratnatunga et al. (2012), Sarokolaei et al. (2013) e Siguenza-Guzman (2014). Nesse rumo, tais autores comentam que o aspecto que melhor caracteriza 0 TDABC é o emprego de equações de tempo, cujo propósito mais relevante é o de evidenciar as atividades mais demoradas e onerosas, além de permitir o uso de vários direcionadores de tempo (as variáveis consideradas nas equações) para determinar o custo de cada atividade.

Acerca dessas equações, Souza, Avelar, Boina e Lara. (2009) afirmam que estas têm origem na identificação das atividades do processo a ser custeado. Por isso, além de identificar as atividades priorizadas, também é necessário fazer a estimativa do 
Custeio Baseado em Atividade e Tempo (TDABC) Aplicado em Lavanderia: Estudo de Caso

Mariélly Warmeling Laucsen Martins, Clarice Flor Marçal, Rodney Wernke, Evandro Bonetti, Letícia

Sandrin

tempo de execução respectivo, que atua como direcionador de custo das atividades. Ressalvam, entretanto, que no TDABC a maior parte das atividades utilizará o consumo de tempo necessário como direcionador, mas não se deve descartar o uso de outros direcionadores (se necessário).

Ainda sobre as equações de tempo, Fachini, Spessatto e Scarpin (2008) salientam que, na execução de um trabalho, pode ser comum o uso de diversas atividades. Com o objetivo de simplificar o modelo, estas diversas atividades podem ser substituídas por uma equação de tempo que representará o tempo gasto nas várias atividades de um serviço ou produto, o que se constitui uma evolução se comparado ao ABC. Outro benefício do TDABC é ser possível computar somente o tempo consumido na execução do trabalho, segregando-o do tempo ocioso do departamento. Dessa forma, se evita a atribuição do custo do período ocioso aos objetos de custeio, o que implicaria custos maiores que aqueles exclusivamente vinculados às atividades consumidas pelos produtos ou serviços. Os citados autores argumentam também que as equações de tempo facilitam a implementação do TDABC nos softwares de gerenciamento, as atualizações posteriores e as projeções de aumento de demanda para as atividades priorizadas.

\subsection{Vantagens e Desvantagens do TDABC}

Em que pesem as características positivas já mencionadas, nesta seção são apresentados alguns benefícios oriundos do TDABC e algumas limitações pertinentes.

No caso dos benefícios, Campanale, Cinquini e Tenucci (2014) apontam a facilidade para implementar e atualizar a ferramenta de custos baseada no TDABC. Em virtude disso, possibilita atualizar as taxas de custo da capacidade prática de cada setor e obter informações sobre o custo dos produtos ou serviços, de forma tempestiva e detalhada, com gastos menores em relação ao que seria necessário despender se utilizado o ABC.

Ratnatunga et al. (2012) e Kaplan (2014) defendem que o TDABC apresenta vantagens em relação ao $A B C$. Acerca disso, registram que o TDABC consegue identificar oportunidades de melhoria; efetuar previsões de gastos de recursos com 
Custeio Baseado em Atividade e Tempo (TDABC) Aplicado em Lavanderia: Estudo de Caso

Mariélly Warmeling Laucsen Martins, Clarice Flor Marçal, Rodney Wernke, Evandro Bonetti, Letícia

Sandrin

determinado tipo de produto ou cliente; aprimorar a eficiência da utilização dos recursos; integrar-se com dados de outros sistemas; ter maior rapidez no processamento dos dados requeridos; e possibilita a utilização de diversas taxas de direcionadores (driver rates) para determinar o custo de cada atividade priorizada.

Por sua vez, Fontoura (2013) afirma que o TDABC traz maior flexibilidade e capacidade de simulações para fundamentar a tomada de decisões. Citam que os defensores dessa forma de custeamento acreditam que sua atualização possa ser trimestral, pois o custo do minuto da atividade só terá mudanças significativas quando houver alteração no nível de eficiência da capacidade ou aumento dos custos fixos.

Além disso, entre os pontos favoráveis, podem ser elencados: simplificação em relação ao $\mathrm{ABC}$; rápida construção do modelo; maior facilidade de integração ou adaptação aos softwares de gestão em uso pelas empresas; possibilidade de evidenciar a utilização da capacidade instalada/ociosa; ser aplicável fora do ambiente fabril; adaptabilidade a contextos empresariais complexos etc. Contudo, ao TDABC podem ser imputadas desvantagens como dificuldade para obter estimativas de tempos exatas e a necessidade de utilizar uma grande base de dados para determinar os tempos de cada atividade executada e suas variações em empresas com muitas etapas produtivas (Pernot, Roodhooft, \& Abbeele, 2007, Anderson, \& Kaplan, 2007, Cardinaels, \& Labro, 2008, Varila, Seppanem, \& Suomala, 2007).

Pereira (2015) salienta que, como o TDABC deixa de considerar a alocação dos custos às atividades, é válido duvidar se realmente se trata de uma evolução perante o $A B C$. Segundo essa autora, o grande diferencial do modelo original eram justamente as duas fases de alocação (antes os recursos eram alocados às atividades e, depois, das atividades para os produtos) que utilizavam critérios de alocação que representavam melhor os consumos de recursos. Ao se abandonar essas características do modelo $A B C$, não se deveria falar em evolução do método, mas considerá-lo uma forma de retrocesso que os seus idealizadores criaram para conseguir "vender" seu novo modelo de custeamento perante as restrições que o $A B C$ sofria.

O mesmo raciocínio é apontado por Gervais, Levant e Ducrocq (2010). Estes entendem que os criadores do TDABC defendem que a principal vantagem desse 
Custeio Baseado em Atividade e Tempo (TDABC) Aplicado em Lavanderia: Estudo de Caso

Mariélly Warmeling Laucsen Martins, Clarice Flor Marçal, Rodney Wernke, Evandro Bonetti, Letícia

Sandrin

método é que proporcionaria uma alternativa para redução da complexidade do custeamento das operações por intermédio das equações de tempo que consideram, de forma simples e menos dispendiosa, questões complexas que afetam os custos. Entretanto, a exatidão das estimativas dos tempos consumidos nas atividades é questionável, visto que recomenda a utilização dos tempos informados pelos funcionários, quando não for possível mensurá-los diretamente. Adicionalmente, os citados autores concluíram que o TDABC se assemelha ao tradicional custeio baseado em padrões e coeficientes de equivalência, ao qual seus idealizadores tentaram adicionar a mensuração da capacidade ociosa.

Por último, De La Villarmois e Levant (2007) registraram que a peculiaridade mais relevante desta forma de custeamento é utilizar apenas um direcionador de custos: o tempo. Com isso, o TDABC seria apenas uma forma de facilitar o uso do $A B C$, cuja simplicidade e baixo custo de uso são atraentes, mas é muitas vezes criticado pela falta de julgamento teórico e de confiabilidade.

\section{METODOLOGIA}

No que tange à metodologia empregada nesta pesquisa, em relação à tipologia quanto aos objetivos, esta pode ser classificada como descritiva, pois referida modalidade visa, segundo Gil (1999), descrever características de determinada população ou fenômeno ou o estabelecimento de relação entre as variáveis. Nessa direção, Andrade (2002) destaca que a pesquisa descritiva se preocupa em observar os fatos, registrá-los, analisá-los, classificá-los e interpretá-los sem a interferência do pesquisador. Pelo aspecto dos procedimentos adotados, a pesquisa caracteriza-se como estudo de caso, de vez que se concentra especificamente numa lavanderia, e suas conclusões limitam-se ao contexto desse objeto de estudo. No âmbito da forma de abordagem do problema, a pesquisa pode ser classificada como "qualitativa", pois é assim que Richardson (1999) denomina os estudos que visam descrever a complexidade de determinado problema, analisar a interação de certas variáveis, compreender e classificar processos dinâmicos vividos por grupos sociais. 
Custeio Baseado em Atividade e Tempo (TDABC) Aplicado em Lavanderia: Estudo de Caso Mariélly Warmeling Laucsen Martins, Clarice Flor Marçal, Rodney Wernke, Evandro Bonetti, Letícia

Sandrin

Quanto à escolha da empresa, esta ocorreu por dois motivos. A primeira razão está ligada à possibilidade de acesso aos dados necessários por parte dos pesquisadores, facultada pelo proprietário do empreendimento. Destarte, a amostra é de caráter intencional, não-probabilística, limitando os resultados do estudo a esse contexto empresarial. A segunda razão para priorizar essa entidade é que esta possui estrutura produtiva com apenas dez setores e trabalhava com mix composto somente por cinco produtos. Essa configuração acarretou maior facilidade na coleta de dados e na elaboração dos cálculos necessários, bem como proporcionou melhores condições de redigir um texto detalhado, abrangendo todos os setores e produtos.

Nesta pesquisa, os procedimentos de coleta de dados foram realizados em outubro e novembro de 2015, abrangendo o contexto de outubro do mesmo ano. Para essa finalidade, inicialmente, foi empregada a técnica de conversas informais (entrevistas não estruturadas) com o gestor da entidade e com o contador. $\mathrm{Na}$ sequência, foi efetuada uma análise documental (nos controles internos e na contabilidade terceirizada) com a intenção de conhecer a situação vigente no que tange aos dados necessários para efetuar o estudo pretendido. Posteriormente, iniciou-se a coleta dos dados requeridos para execução do trabalho nos controles internos existentes, além de outros informes mais específicos que foram obtidos junto ao gerente e ao contador da firma.

Ainda, no que tange aos aspectos formais e no sentido de avaliar o rigor metodológico deste estudo de caso, foram utilizados os parâmetros recomendados por Marques, Camacho e Alcantara (2015):

a) Quanto ao objeto de estudo: procurou-se entender o fenômeno proposto em seu contexto real; explicou-se o motivo de adotar esta estratégia de pesquisa; há uma ligação entre o fenômeno em questão e o contexto da pesquisa; a pergunta de pesquisa está claramente formulada, e o tipo de estudo está evidenciado (descritivo).

b) Quanto à coleta de dados: verifica-se a existência de múltiplas formas de evidenciação (entrevistas com gestor e contador, controles internos, contabilidade etc.) que permitem a triangulação dos dados; é possível atestar a confiabilidade dos dados pelos controles internos e contábeis utilizados; medidas operacionais (como custo 
Custeio Baseado em Atividade e Tempo (TDABC) Aplicado em Lavanderia: Estudo de Caso

Mariélly Warmeling Laucsen Martins, Clarice Flor Marçal, Rodney Wernke, Evandro Bonetti, Letícia

Sandrin

unitário e nível de ociosidade) foram evidenciadas para validar o construto do estudo; há explicação sobre a forma como os dados foram obtidos e há a possibilidade de replicar a coleta de dados em outro contexto.

c) Quanto à análise dos dados: os resultados da pesquisa refletem os dados coletados, e houve uso de teoria anterior para embasar as análises.

d) Quanto aos resultados: foram relatadas contribuições na geração do conhecimento em relação a estudos precedentes e ainda foram feitos alertas para pontos que ainda precisam de continuação nesse tipo de investigação (mencionados em seção posterior).

Os procedimentos executados para realizar a pesquisa são apresentados de forma detalhada nos itens seguintes.

\section{APRESENTAÇÃO DOS DADOS E ANÁLISE DOS RESULTADOS}

O foco da pesquisa foi uma lavanderia situada no município de Laguna (SC), cujo nome foi omitido por solicitação do proprietário. Em outubro e novembro de 2015, essa empresa poderia ter sido caracterizada como pequena empresa, visto que contava com apenas dois funcionários sob o comando do dono do empreendimento.

A dificuldade para suportar o crescimento da concorrência e a compreensão do proprietário de que deveria melhorar a forma de conduzir a lavanderia foram os dois principais aspectos que contribuíram para que o estudo fosse realizado. Conforme o proprietário da lavanderia, a dificuldade maior estava relacionada com o custeamento do trabalho necessário para executar cada serviço comercializado. Em razão disso, foi sugerido, pelos pesquisadores, implementar uma planilha de custos pelo TDABC, adaptada ao contexto citado, conforme descrito nos próximos tópicos. Convém destacar ainda que não se tratou de consultoria, visto que se fundamentou em trabalho de conclusão de curso de uma das autoras deste artigo. 
Custeio Baseado em Atividade e Tempo (TDABC) Aplicado em Lavanderia: Estudo de Caso

Mariélly Warmeling Laucsen Martins, Clarice Flor Marçal, Rodney Wernke, Evandro Bonetti, Letícia

Sandrin

\subsection{Levantamento dos Custos Indiretos Mensais dos Setores}

A primeira etapa para determinar o custo pelo TDABC consistiu em realizar o levantamento de quanto custa (em $\mathrm{R} \$$ ) manter a estrutura do empreendimento capaz de executar as atividades da área produtiva. Nesse sentido, as operações da empresa pesquisada requeriam o consumo de recursos relacionados com os itens a seguir:

a) Depreciação do maquinário: para apurar esse valor, foi utilizado o conceito de "depreciação econômica", ou seja, o valor monetário $(R \$)$ de cada equipamento dividido pelo número de meses previstos para vida útil. No caso em tela, totalizou $R \$$ 1.354,49 por mês;

b) Aluguel predial: o valor do aluguel mensal do prédio utilizado pela lavanderia é de $\mathrm{R} \$ 3.400,00$ e esse montante foi rateado entre os setores em proporção à metragem quadrada ocupada;

c) Salários e encargos sociais: a partir da folha de pagamentos fornecida pela contabilidade da empresa, foi apurado que o valor mensal equivale a $R \$ 6.005,00$. Os valores atribuídos aos setores levaram em conta estimativas do tempo despendido mensalmente pelos funcionários em cada setor enfocado no estudo;

d) Energia elétrica: o valor da fatura mensal de energia elétrica $(R \$ 1.402,37)$ foi distribuído entre os setores com base na estimativa de consumo em termos de $\mathrm{kW} / \mathrm{hora}$ do maquinário em cada um dos setores, conforme o equipamento utilizado nos mesmos;

e) Manutenção de máquinas: os gastos dos setores foram estimados em razão do tempo médio despendido com cada equipamento pelo mecânico terceirizado da companhia, que cobrava $\mathrm{R} \$ 700,00$ por mês para realizar os serviços pertinentes.

Então, a partir dessas fontes, foi elaborada a Tabela 1, onde os valores mensais foram divididos pelo expediente mensal de 198 horas para apurar o custo por hora $(R \$)$ de cada setor. 
Custeio Baseado em Atividade e Tempo (TDABC) Aplicado em Lavanderia: Estudo de Caso

Mariélly Warmeling Laucsen Martins, Clarice Flor Marçal, Rodney Wernke, Evandro Bonetti, Letícia

Sandrin

Tabela 1

Custo por hora dos setores

\begin{tabular}{|c|c|c|c|c|c|c|c|c|}
\hline Itens & $\begin{array}{l}\text { Deprec. } \\
\text { Máq.R\$ }\end{array}$ & $\begin{array}{c}\text { Aluguel } \\
\text { Pred. } R \$\end{array}$ & $\begin{array}{l}\text { Salários e } \\
\text { encarg. } R \$\end{array}$ & $\begin{array}{l}\text { Energia } \\
\text { elétr.R\$ }\end{array}$ & $\begin{array}{l}\text { Manut. } \\
\text { Máq.R\$ }\end{array}$ & $\begin{array}{l}\text { a) Custo do } \\
\text { mês } R \$\end{array}$ & $\begin{array}{l}\text { b) Exped. } \\
\text { do mês (h) }\end{array}$ & $\begin{array}{l}\mathrm{C}=\mathrm{a} / \mathrm{b}) \text { Custc } \\
\text { por hora } \mathrm{R} \$\end{array}$ \\
\hline Entr.Pc./Micro+lmpress. & 155,72 & 321,11 & $1.651,07$ & 28,91 & 70,00 & $2.226,81$ & 198 & 11,2465 \\
\hline Entr.Pc./Balança Dig. & 27,08 & 11,06 & 505,41 & 0,41 & 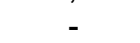 & 543,96 & 198 & 2,7473 \\
\hline Manual/Sep. Peças & - & 594,02 & 270,00 & - & - & 864,02 & 198 & 4,3637 \\
\hline Lavagem/Lavadora & 475,00 & 290,15 & 405,00 & 142,48 & 301,00 & $1.613,63$ & 198 & 8,1497 \\
\hline Secagem/Secadora & 208,33 & 280,42 & 405,00 & 706,48 & 119,00 & $1.719,23$ & 198 & 8,6830 \\
\hline Passadoria/Mesa de passar & 180,21 & 453,81 & $1.080,00$ & 497,58 & 105,00 & $2.316,59$ & 198 & 11,7000 \\
\hline Embalag./Embal.Roupas & 52,08 & 132,69 & 270,00 & 13,49 & 35,00 & 503,26 & 198 & 2,5417 \\
\hline Armaz./Cabideiro Eletr. & 120,50 & 537,84 & 67,50 & 8,51 & 70,00 & 804,35 & 198 & 4,0624 \\
\hline Armaz./Manual-Prat.MDF & 69,44 & 457,79 & 202,50 & - & - & 729,73 & 198 & 3,6855 \\
\hline Saída Pç./Micro-comp. & 66,11 & 321,11 & $1.148,52$ & 4,52 & - & $1.540,27$ & 198 & 7,7792 \\
\hline Totais & $1.354,49$ & $3.400,00$ & $6.005,00$ & $1.402,37$ & 700,00 & $12.861,86$ & & \\
\hline
\end{tabular}

Nota. Fonte: Elaborado pelos autores

Com base nos valores da Tabela 1, é possível perceber que há duas atividades/setores que se destacam em termos dos valores de custo por hora e de gasto total mensal: "Passadoria/Mesa de passar" $(R \$ 2.316,59$ por mês e $R \$$ 11,70/hora) e "Entr. Pç/Micro+Impress." (R \$ 2.226,81 por mês e $R \$ 11,25 /$ hora).

Além destes dois, cabe destacar o custo por hora dos setores/atividades "Secagem/Secadora" ( $R \$$ 8,68), "Lavagem/Lavadora" ( $R \$ 8,15)$ e "Saída Pç/Microcomp." ( $R \$ 7,78)$, enquanto que os demais possuem custo/hora inferior a $R \$ 4,50$.

\subsection{Capacidade Prática dos Setores e Taxa de Custos de Capacidade Prática por Minuto}

Para aplicar o método de custeio TDABC no presente estudo, também foi necessário calcular a taxa do custo da capacidade prática, por minuto, dos referidos setores.

Nesse rumo, calculou-se o expediente mensal em minutos (198 horas de expediente mensal X 60 minutos), onde se constatou a disponibilidade de 11.880 minutos por setor. Dividindo-se o custo total mensal do setor (atribuído na Tabela 1) pela respectiva capacidade do expediente de trabalho do mês, foi calculada a "taxa do 
Custeio Baseado em Atividade e Tempo (TDABC) Aplicado em Lavanderia: Estudo de Caso Mariélly Warmeling Laucsen Martins, Clarice Flor Marçal, Rodney Wernke, Evandro Bonetti, Letícia

custo da capacidade prática (em R\$ /min.)" dessas unidades da empresa, conforme evidenciado na Tabela 2.

Tabela 2

Taxa do custo de capacidade prática do setor (TDABC)

\begin{tabular}{ccccc}
\hline & $\begin{array}{c}\text { Expediente } \\
\text { Mensal } \\
\text { (horas) }\end{array}$ & $\begin{array}{c}\text { Expediente } \\
\text { Mensal } \\
\text { Iminutos) }\end{array}$ & $\begin{array}{c}\text { Custo total } \\
\text { Mensal } \\
(\mathrm{R} \$)\end{array}$ & $\begin{array}{c}\text { Taxa do custo } \\
\text { de capacidade } \\
\text { prática }(\mathrm{R} \$ / \mathrm{min} .)\end{array}$ \\
\hline Entr.Pç./Micro+Impress. & 198 & 11.880 & $2.226,81$ & 0,187442 \\
Entr.Pç./Balança Dig. & 198 & 11.880 & 543,96 & 0,045788 \\
Manual/Sep. Peças & 198 & 11.880 & 864,02 & 0,072729 \\
Lavagem/Lavadora & 198 & 11.880 & $1.613,63$ & 0,135828 \\
Secagem/Secadora & 198 & 11.880 & $1.719,23$ & 0,144716 \\
Passadoria/Mesa de passar & 198 & 11.880 & $2.316,59$ & 0,194999 \\
Embalag./Embal.Roupas & 198 & 11.880 & 503,26 & 0,042362 \\
Armaz./Cabideiro Eletr. & 198 & 11.880 & 804,35 & 0,067706 \\
Armaz./Manual-Prat.MDF & 198 & 11.880 & 729,73 & 0,061425 \\
Saída Pç./Micro-comp. & 198 & 11.880 & $1.540,27$ & 0,129653 \\
Totais & 1.980 & 118.800 & $12.861,86$ & - \\
\hline
\end{tabular}

Nota. Fonte: Elaborado pelos autores

Nos dados da Tabela 2, destacam-se as taxas de custo de capacidade prática dos setores "Entr.Pç./Micro+Impress.", com R\$ 0,187 por minuto, e "Passadoria/Mesa de passar", com $\mathrm{R} \$ 0,195$ por minuto.

\subsection{Determinação do Custo Unitário e Total de cada Produto}

A etapa seguinte do método TDABC visa determinar o custo unitário de cada produto ou serviço prestado pela empresa, cujos procedimentos utilizados no caso em lume estão representados sinteticamente na Tabela 3 e descritos com detalhes em seguida. 
Custeio Baseado em Atividade e Tempo (TDABC) Aplicado em Lavanderia: Estudo de Caso

Mariélly Warmeling Laucsen Martins, Clarice Flor Marçal, Rodney Wernke, Evandro Bonetti, Letícia

Sandrin

Tabela 3

Custo unitário e total por produto pelo TDABC

\begin{tabular}{|c|c|c|c|c|c|c|}
\hline Itens & Edred-pç & Camis-pç & Terno-pç & Roup/Pas.-kg & Roup/S/Pas.-kg & Totais \\
\hline \multicolumn{7}{|c|}{ a) Produção por hora (em unidades) } \\
\hline Entr.Pç./Micro+Impress. & 30 & 30 & 30 & 12 & 12 & - \\
\hline $\begin{array}{c}\text { Entr.Pç./Balança Dig. } \\
\text { Outros... }\end{array}$ & - & - & - & 180 & 180 & - \\
\hline \multicolumn{7}{|c|}{ b) Quantidade por minuto (a/60') } \\
\hline Entr.Pç./Micro+Impress. & 0,5000 & 0,5000 & 0,5000 & 0,2000 & 0,2000 & - \\
\hline $\begin{array}{l}\text { Entr.Pç./Balança Dig. } \\
\text { Outros... }\end{array}$ & - & - & - & 3,0000 & 3,0000 & - \\
\hline \multicolumn{7}{|c|}{ c) Minutos por unidade produzida (1/b) } \\
\hline Entr.Pç./Micro+Impress. & 2,0000 & 2,0000 & 2,0000 & 5,0000 & 5,0000 & - \\
\hline $\begin{array}{c}\text { Entr.Pç./Balança Dig. } \\
\text { Outros... }\end{array}$ & - & - & - & 0,3333 & 0,3333 & - \\
\hline \multicolumn{7}{|c|}{ d) Taxa do custo de capacidade do setor ( $\mathrm{R} \$ / \mathrm{min}$.) } \\
\hline Entr.Pç./Micro+Impress. & 0,1874 & 0,1874 & 0,1874 & 0,1874 & 0,1874 & - \\
\hline $\begin{array}{l}\text { Entr.Pç./Balança Dig. } \\
\text { Outros... }\end{array}$ & 0,0458 & 0,0458 & 0,0458 & 0,0458 & 0,0458 & - \\
\hline \multicolumn{7}{|c|}{ e) $=c X d)$ Custo unitário $(R \$)$} \\
\hline Entr.Pç./Micro+Impress. & 0,3749 & 0,3749 & 0,3749 & 0,9372 & 0,9372 & - \\
\hline $\begin{array}{c}\text { Entr.Pç./Balança Dig. } \\
\text { Outros... }\end{array}$ & - & - & - & 0,0153 & 0,0153 & - \\
\hline Total do custo unit. por serviço & 3,9249 & 1,6070 & 2,2991 & 2,8640 & 1,9863 & - \\
\hline f) Quantidade produzida/mês & 295 & 413 & 166 & 374 & 292 & 1.540 \\
\hline \multicolumn{7}{|c|}{ g)=eXf) Custo total atribuído no período $(\mathrm{R} \$)$} \\
\hline Entr.Pç./Micro+Impress. & 110,59 & 154,83 & 62,23 & 350,52 & 273,67 & 951,83 \\
\hline $\begin{array}{c}\text { Entr.Pç./Balança Dig. } \\
\text { Outros... }\end{array}$ & - & - & - & 5,71 & 4,46 & 10,16 \\
\hline Total de custo atrib. ao serviço & $1.157,85$ & 663,69 & 381,66 & $1.071,14$ & 580,00 & $3.854,34$ \\
\hline
\end{tabular}

Nota. Fonte: Elaborado pelos autores

Como visto, na Tabela 3 estão expostos os valores listados somente em relação aos dois primeiros setores, enquanto os demais estão "representados" na linha "Outros...", por restrição de espaço no artigo. Contudo, para esclarecer acerca dos procedimentos mencionados na Tabela 3, utiliza-se como exemplo a rotina de cálculo empregada no caso do produto "Edred-pç", em que foram percorridas as seguintes etapas: 
Custeio Baseado em Atividade e Tempo (TDABC) Aplicado em Lavanderia: Estudo de Caso

Mariélly Warmeling Laucsen Martins, Clarice Flor Marçal, Rodney Wernke, Evandro Bonetti, Letícia

Sandrin

a) Produção por hora (em unidades) - conforme estimativa do gestor da lavanderia, seria possível manusear 30 unidades por hora desse produto no setor "Entr.Pç./Micro+Impress.";

b) Quantidade por minuto (item "a" / 60 minutos) -Para apurar esse volume por minuto, as 30 peças por hora foram divididas por 60 minutos, o que equivale a 0,50 unidade por minuto;

c) Minutos por unidade produzida (1/b) - Nessa fase, divide-se "1" pela quantidade por minuto apurada no item "b", que, neste caso do "Edred-pç", era 0,50. Com isso, chega-se ao tempo estimado de 2 minutos por unidade;

d) Taxa do custo de capacidade do setor ( $\mathrm{R} \$ / \mathrm{min}$.) -O valor que cabe a cada setor foi calculado, anteriormente, na Tabela 1. No contexto do exemplo, o setor

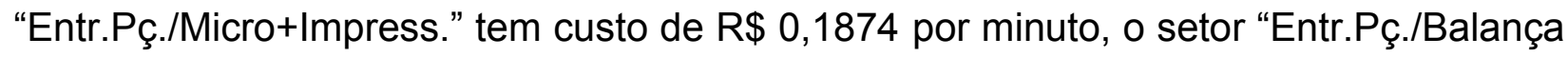
Dig." custa $\mathrm{R} \$ 0,0458$, e assim por diante com os demais setores;

e) Custo unitário $(R \$)$ - Nessa parte da Tabela 3, o cálculo requer a multiplicação do tempo (em minutos) que o produto consome do setor (item "c") pela taxa do custo de capacidade respectivo (item "d"). Se uma unidade do produto "Edredpç" consome 2 minutos do setor "Entr.Pç./Micro+Impress." para ser processada e cada minuto neste custa $R \$ 0,1874$, é cabível considerar que o tempo total despendido custaria $R \$ 0,3749$ ( $R \$ 0,1874 \times 2$ minutos). O mesmo vale para os demais setores que esse item percorria para ser concluído. Com isso, chegou-se ao valor de $\mathrm{R} \$ 3,9249$ como custo unitário para o produto citado;

f) Quantidade produzida/mês - Com base nos controles internos do mês pesquisado, foram lavadas 295 unidades do "Edred-pç", 413 unidades do "Camis-pç", 166 unidades do "Terno-pç", 374 quilos do "Roup/Pas.-kg" e 292 quilos do "Roup/S/Pas.-kg". Ou seja, o total da produção do período foi 1.540 unidades ou quilos (conforme a praxe adotada na empresa para distinguir os volumes trabalhados a partir do tipo de roupa lavada);

g) Custo total atribuído no período $(R \$)$ - Para chegar aos valores totais alocados aos produtos ao término do período, bastou multiplicar o custo unitário (apurado na etapa "e") pela quantidade produzida no mês (registrada na parte "f" da 
Custeio Baseado em Atividade e Tempo (TDABC) Aplicado em Lavanderia: Estudo de Caso Mariélly Warmeling Laucsen Martins, Clarice Flor Marçal, Rodney Wernke, Evandro Bonetti, Letícia

Sandrin

Tabela 3). No caso do "Edred-pç", as 295 unidades acarretaram custo total de $R \$$ 110,59 somente no setor "Entr.Pç./Micro+Impress.". Ao somarem-se os demais setores que esse produto utilizou, atingiu-se o total de $R \$ 1.157,85$ para as 295 unidades do período. Por outro ângulo, também foi possível conhecer os valores totais despendidos com cada setor. Nessa direção, somando-se todos os valores dos produtos que passaram pelo setor "Entr.Pç./Micro+Impress.", chegou-se ao montante de $\mathrm{R} \$$ 951,83 (última coluna da parte "g" da tabela citada). Adicionando-se os valores totais dos outros setores, a metodologia utilizada permitiu alocar $R \$ 3.854,34$ dos custos do mês, o que representa $29,97 \%$ do custo total mensal (conforme expresso, anteriormente, na Tabela 1).

A alocação de apenas uma parcela dos custos do período está atrelada à existência de capacidade ociosa, conforme comentado na próxima seção.

\subsection{Capacidade Instalada, Utilizada e Ociosa do Período}

Um dos principais benefícios do método de custeio TDABC reside na identificação dos níveis das capacidades de produção "instalada", "utilizada" e "ociosa" dos setores, conforme perceptível nas Tabelas 4 e 5 explicadas adiante.

Para chegar a essas informações, o primeiro passo consiste em determinar o consumo de minutos de cada setor (ou atividade). Com esse propósito, basta multiplicar (I) quantidade de minutos consumida por produto (já determinada no item "c" da Tabela 3 da seção precedente) pelo (ii) volume processado no mês enfocado (mencionado na parte "f" da Tabela 3). Por exemplo, no caso do produto "Edred-pç", as 295 unidades do período consumiram 2 minutos cada uma no setor "Entr.Pç./Micro+Impress.", o que implicou o consumo de 590,00 minutos deste setor no mês da pesquisa somente com esse item. Os resultados dessa multiplicação para os demais produtos e setores estão destacados na Tabela 4. 
Custeio Baseado em Atividade e Tempo (TDABC) Aplicado em Lavanderia: Estudo de Caso Mariélly Warmeling Laucsen Martins, Clarice Flor Marçal, Rodney Wernke, Evandro Bonetti, Letícia

Sandrin

Tabela 4

Consumo de minutos pela produção do mês

\begin{tabular}{ccccccc}
\hline Itens & Edred-pç & Camis-pç & Terno-pç & Roup/Pas.-kg & Roup/S/Pas.-kg & Totais \\
\hline Entr.Pç./Micro+Impress. & 590,0 & 826,0 & 332,00 & $1.870,00$ & $1.460,00$ & $5.078,00$ \\
Entr.Pç./Balança Dig. & - & - & - & 124,67 & 97,33 & 222,00 \\
Manual/Sep. Peças & 442,5 & 413,0 & 249,00 & $1.122,00$ & 876,00 & $3.102,50$ \\
Lavagem/Lavadora & $4.425,0$ & 354,0 & 276,67 & $1.122,00$ & 876,00 & $7.053,67$ \\
Secagem/Secadora & $2.212,5$ & 5,9 & 7,69 & 561,00 & 438,00 & $3.225,09$ \\
Passadoria/Mesa de passar & - & $1.652,0$ & $1.106,67$ & $1.683,42$ & - & $4.442,09$ \\
Embalag./Embal.Roupas & 885,0 & 619,5 & 332,00 & - & - & $1.836,50$ \\
Armaz./Cabideiro Eletr. & - & 413,0 & 166,00 & - & - & 579,00 \\
Armaz./Manual-Prat.MDF & 295,0 & - & - & 374,00 & 292,00 & 961,00 \\
Saída Pç./Micro-comp. & 295,0 & 413,0 & 166,00 & 374,00 & 292,00 & $1.540,00$ \\
Totais & $9.145,0$ & $4.696,4$ & $2.636,02$ & $7.231,09$ & $4.331,33$ & $28.039,84$ \\
\hline
\end{tabular}

Nota. Fonte: Elaborado pelos autores

Como visto na Tabela 4, a produção do período gerou consumo de 5.078 minutos no setor "Entr.Pç./Micro+Impress.", 222 minutos no setor "Entr.Pç./Balança Dig." e assim por diante com as demais unidades da empresa em tela. Destarte, o referido volume produzido (1.540 peças ou quilos, a depender do produto manuseado, conforme citado na Tabela 3 da seção anterior), totalizou 28.039,84 minutos de capacidade produtiva "utilizada" efetivamente no mês do estudo em termos do conjunto de setores abrangidos.

A partir dessa informação relativa à capacidade utilizada, o TDABC faculta o conhecimento da ociosidade da capacidade instalada, como descrito na Tabela 5. 
Custeio Baseado em Atividade e Tempo (TDABC) Aplicado em Lavanderia: Estudo de Caso

Mariélly Warmeling Laucsen Martins, Clarice Flor Marçal, Rodney Wernke, Evandro Bonetti, Letícia

Sandrin

Tabela 5

Ociosidade em minutos, percentual e valor monetário

\begin{tabular}{cccccc}
\hline Itens & $\begin{array}{c}\text { Expediente } \\
\text { mensal (min.) }\end{array}$ & $\begin{array}{c}\text { Consumo de } \\
\text { min./mês }\end{array}$ & $\begin{array}{c}\text { Ociosidade } \\
\text { Mensal (min.) }\end{array}$ & $\begin{array}{c}\text { Ociosidade } \\
\text { Mensal }(\%)\end{array}$ & $\begin{array}{c}\text { Ociosidade } \\
\text { Mensal (R\$) }\end{array}$ \\
\hline Entr.Pç./Micro+Impress. & 11.880 & $5.078,00$ & $6.802,00$ & $57,26 \%$ & $1.274,98$ \\
Entr.Pç./Balança Dig. & 11.880 & 222,00 & $11.658,00$ & $98,13 \%$ & 533,79 \\
Manual/Sep. Peças & 11.880 & $3.102,50$ & $8.777,50$ & $73,88 \%$ & 638,38 \\
Lavagem/Lavadora & 11.880 & $7.053,67$ & $4.826,33$ & $40,63 \%$ & 655,55 \\
Secagem/Secadora & 11.880 & $3.225,09$ & $8.654,91$ & $72,85 \%$ & $1.252,51$ \\
Passadoria/Mesa de passar & 11.880 & $4.442,09$ & $7.437,91$ & $62,61 \%$ & $1.450,39$ \\
Embalag./Embal. Roupas & 11.880 & $1.836,50$ & $10.043,50$ & $84,54 \%$ & 425,47 \\
Armaz./Cabideiro Eletr. & 11.880 & 579,00 & $11.301,00$ & $95,13 \%$ & 765,15 \\
Armaz./Manual-Prat. MDF & 11.880 & 961,00 & $10.919,00$ & $91,91 \%$ & 670,70 \\
Saída Pç./Micro-comp. & 11.880 & $1.540,00$ & $10.340,00$ & $87,04 \%$ & $1.340,61$ \\
Totais & 118.800 & $28.039,84$ & $90.760,16$ & $76,40 \%$ & $9.007,52$ \\
\hline
\end{tabular}

Nota. Fonte: Elaborado pelos autores

Como destacado na Tabela 2 (seção 4.2), o expediente mensal de minutos era de 11.880 em cada setor e, na Tabela 4, foi apurado o consumo de minutos dos setores no mês da pesquisa. Nesse caso, a subtração da (I) capacidade utilizada (em minutos) da (ii) capacidade instalada ou do expediente disponível (em minutos) fornece o (iii) nível de ociosidade (em minutos) dos setores. Exegese: no âmbito do setor "Entr.Pç./Micro+Impress.", dos 11.880 minutos disponíveis, foram efetivamente consumidos pela produção cerca de 5.078 minutos. Com isso, aproximadamente 6.802 minutos ficaram ociosos naquele setor (coluna 4 da Tabela 5). No que concerne ao total da empresa, dos 118.800 minutos de capacidade instalada, foram consumidos 28.039,84 minutos, acarretando 90.760,16 minutos de ociosidade.

Uma forma de aprimorar e facilitar a análise do gestor consiste em mensurar o percentual de ociosidade como está delineado na coluna 5 da Tabela 5 . Ou seja, os $90.760,16$ minutos ociosos equivalem a $76,40 \%$ da capacidade instalada total de produção da empresa $(90.760,16$ / $118.800 \times 100)$. Por esse prisma, os maiores níveis de ociosidade foram identificados nos setores na seguinte ordem decrescente: "Entr.Pç./Balança Dig." (98,13\%), "Armaz./Cabideiro Eletr." (95,13\%), "Armaz./ManualPrat.MDF" (91,91\%), "Saída Pç./Micro-comp." (87,04\%), "Embalag./Embal.Roupas" 
Custeio Baseado em Atividade e Tempo (TDABC) Aplicado em Lavanderia: Estudo de Caso

Mariélly Warmeling Laucsen Martins, Clarice Flor Marçal, Rodney Wernke, Evandro Bonetti, Letícia

Sandrin

(84,54\%), "Manual/Sep. Peças" (73,88\%), "Secagem/Secadora" (72,85\%), "Passadoria/Mesa de passar" (62,61\%), “Entr.Pç./Micro+Impress." (57,26\%) e "Lavagem/Lavadora" (40,63\%).

Contudo, seria mais relevante a análise da ociosidade no que tange aos valores monetários que the podem ser associados e que, no mês em lume, totalizou $R \$$ 9.007,52 na lavanderia em estudo. Para chegar a esse valor (vide última coluna da Tabela 5), foi necessário multiplicar a quantidade de minutos ociosos em cada setor pela respectiva "taxa do custo de capacidade prática $(\mathrm{R} \$ / \mathrm{min}$.)", que havia sido calculada na Tabela 2 (seção 4.2). Por exemplo: como o setor "Entr.Pç./Micro+Impress." ficou 6.802 minutos ociosos, e o custo por minuto neste foi de $R \$ 0,187442$, o valor total da ociosidade chegou a $R \$ 1.274,98$ (6.802 minutos $X R \$$ $0,187442)$.

Ao avaliar-se por esse parâmetro monetário, se conhece um cenário bem distinto em termos de ordem decrescente de ociosidade por setores na comparação com o percentual de tempo ocioso de cada setor. Nesse rumo, o setor "Passadoria/Mesa de passar" ficaria na primeira posição, com $\mathrm{R} \$ 1.450,39$ de ociosidade (mas foi o oitavo colocado no critério anterior), e o setor "Saída Pç./Micro-comp." ocuparia a segunda colocação, com ociosidade mensal de $R \$ 1.340,61$ (situava-se na quarta posição em termos do percentual de ociosidade).

No caso do setor "Entr.Pç./Micro+Impress.", sua ociosidade atingiu $R \$ 1.274,98$ e foi o terceiro maior valor no mês em tela (era o nono colocado no ranking anterior), enquanto que o setor "Secagem/Secadora" acumulou ociosidade equivalente a R\$ 1.252,51 (quarto maior valor do período, mas ficou na sétima posição no percentual de ociosidade). Os demais setores abrangidos no estudo tiveram níveis de ociosidade menos representativos: "Armaz./Cabideiro Eletr." R \$ 765,15; "Armaz./Manual-Prat.MDF" R\$ 670,70; "Lavagem/Lavadora" R\$ 655,55; "Manual/Sep. Peças" R\$ 638,38; "Entr.Pç./Balança Dig." R \$ 533,79 e “Embalag./Embal.Roupas" R \$ 425,47.

Ao conhecer esses números acerca da ociosidade, o gestor da lavanderia considerou-os aceitáveis, tendo em vista o momento econômico vivenciado na ocasião 
Custeio Baseado em Atividade e Tempo (TDABC) Aplicado em Lavanderia: Estudo de Caso

Mariélly Warmeling Laucsen Martins, Clarice Flor Marçal, Rodney Wernke, Evandro Bonetti, Letícia

Sandrin

da pesquisa, especialmente pela queda acentuada nas vendas em relação aos anos 2013 e 2014.

\section{CONSIDERAÇÕES FINAIS}

Esta pesquisa pretendeu responder à questão sobre como utilizar o TDABC para determinar o custo da prestação dos serviços de lavanderia de pequeno porte, tendo como objetivo de estudo calcular o custo dos serviços prestados por intermédio desse método. Acerca disso, os autores consideram que atingiram tal objetivo de vez que foi calculado o custo de prestação dos serviços da empresa em lume conforme descrito nas seções precedentes. Portanto, a questão levantada pôde ser convenientemente respondida, pois restou evidenciada a possibilidade de aplicação do TDABC na lavanderia em tela com base nos pressupostos da metodologia criada por Kaplan e Anderson (2004).

Como resultados do estudo, merecem ser destacados alguns aspectos. $O$ primeiro refere-se à possibilidade de mensurar, com relativa facilidade, o custo de prestar o serviço de lavação para os produtos abrangidos, conforme disposto na Tabela 3. Nesse sentido, foi apurado o custo unitário e o total para cada um dos cinco tipos de produtos enfocados, o que permitiu identificar os itens que mais consumiram custos no período. Por exemplo, em termos unitários, a lavação de cada unidade comercializada (peça ou quilo, conforme o caso) custava valores bem distintos: "Edred-pç" R\$3,92; "Camisa-pç" R\$ 1,61; "Terno-pç" R\$ 2,30; "Roup/Pas.-kg” R\$ 2,86 e "Roup/S/Pas.-kg" $\mathrm{R} \$ 1,99$ (valores arredondados).

Conhecidos o custo unitário e o volume mensal processado, também foi possível identificar o quanto cada item consumiu ao final do período. Nesse caso, do custo total mensal de $R \$ 12.861,86$ (Tabela 1 ) foi alocado aos produtos o montante de $R \$$ 3.854,34 (vide última linha da Tabela 3).

Essa parcela não alocada representa a ociosidade da capacidade produtiva instalada, cujos tempos e valores respectivos de cada setor envolvido do estudo estão descritos na Tabela 5. Ou seja, do expediente disponível de 118.800 minutos, foram 
Custeio Baseado em Atividade e Tempo (TDABC) Aplicado em Lavanderia: Estudo de Caso

Mariélly Warmeling Laucsen Martins, Clarice Flor Marçal, Rodney Wernke, Evandro Bonetti, Letícia

Sandrin

consumidos na produção 28.039,84 minutos, acarretando ociosidade de 90.760,16 minutos $(76,40 \%)$. Para esse nível de capacidade não utilizada, foi atribuído o valor total de custos da ordem de $R \$ 9.007,52$. Portanto, foi possível segregar a parcela dos custos efetivamente consumida $(R \$ 3.854,34)$ daquela que ficou ociosa $(R \$ 9.007,52)$ no mês.

O terceiro aspecto a salientar como relevante é que permitiu conhecer o custo por minuto em cada uma das etapas do processo produtivo da lavanderia. Então, a título de exemplo, é pertinente citar que, para o setor "Entr.Pç./Micro+Impress.", a taxa do custo de capacidade por minuto foi calculada em $R \$ 0,19$, enquanto que, no "Entr.Pç./Balança Dig.", esse mesmo valor foi de apenas $\mathrm{R} \$ 0,05$. Esses dois casos atestam a importância de conhecer o custo efetivo de cada setor do negócio de forma tecnicamente consistente, como permitido pelo TDABC, tanto do ponto de vista da priorização de ações quanto da precificação dos produtos.

Para finalizar, é pertinente ressalvar que um estudo de caso, por sua natureza, circunscreve as conclusões oriundas ao âmbito da entidade pesquisada. Portanto, os achados desta se limitam a esse contexto empresarial. Contudo, os procedimentos adotados e a descrição efetuada ao longo do texto permitem que pesquisas assemelhadas posteriormente sejam aplicadas a outros contextos empresariais. Por isso, recomenda-se que futuros estudos abordem esse tema em empresas de outros segmentos e portes, a fim de corroborar ou negar os achados relatados neste artigo.

\section{REFERÊNCIAS}

Andrade, M. M. de (2002). Como preparar trabalhos para cursos de pós-graduação: noções práticas. São Paulo: Atlas.

Baldvinsdottir, G., Mitchell, F., \& Nørreklit, H. (2010).Issues in the relationship between theory and practice in management accounting. Management Accounting Research, $21(2), 79-82$.

Barret, R. (2005). Time-Driven Costing: the bottom line on the new ABC. Business Performance

Management.

Recuperado

de: 
Custeio Baseado em Atividade e Tempo (TDABC) Aplicado em Lavanderia: Estudo de Caso

Mariélly Warmeling Laucsen Martins, Clarice Flor Marçal, Rodney Wernke, Evandro Bonetti, Letícia

Sandrin

http://businessfinancemag.com/business-performance-management/time-drivencosting-bottom-line-new-abc.

Baxendale, S. J. (2001). Activity-based Costing for the small business: a primer. Business Horizons, 4(1), 61-68. doi: https://doi.org/10.1016/S0007-6813(01)800100 .

Campanale, C., Cinquini, L., \& Tenucci, A. (2014). Time-driven activity-based costing to improve transparency and decision making in healthcare: a case study. Qualitative Research in Accounting \& Management, 11(2), 165-186.

Cardinaels, E., \& Labro, E. (2008). On the determinants of measurement error in Timedriven Costing. The Accounting Review, 83(3), 735-756.

Dalci, I., Tanis, V., \& Kosan, L. (2010). Customer profitability analysis with time-driven activity-based costing: a case study in a hotel. International Journal of Contemporary Hospitality Management, 22(5), 609-637. doi: http://dx.doi.org.ez222.periodicos.capes.gov.br/10.1108/09596111011053774

Dalmácio, F. Z., Rezende, A. J., \& Aguiar, A. B. (2007). Uma aplicação do Time-Driven ABC Model no setor de serviço hospitalar: a nova abordagem ABC proposta por Kaplan e Anderson. Contabilidade Vista \& Revista, 18(2), 11-34.

De La Villarmois, O., \& Levant, Y. (2007). Le time-driven ABC: la simplification de l'évaluation des coûts par le recours aux équivalents - un essai de positionnement. Finance Contrôle Stratégie, 10(1), 149-182.

De Souza, A. A., Avelar, E. A., Boina, T. M., \& Lara, C. O. (2009, Julho). Análise dos estudos empíricos realizados sobre o time-driven ABC entre os anos de 2004 e 2008. Anais do Congresso USP de Controladoria e Contabilidade, São Paulo, SP, Brasil, 9.

Dorneles, F. T. (2004). Proposta de sistemática informatizada de custeio para apoio à decisão em pequenas e médias empresas industriais Dissertação de Mestrado). Universidade Federal do Rio Grande do Sul -UFRGS, Porto Alegre, RS, Brasil.

Everaert, P., \& Bruggeman,W. (2007). Time-driven activity-based costing: exploring the underlying model. Journal of Cost Management, 21(2), 16-20.

Everaert, P., Bruggeman, W., Sarens, G., Anderson, S. R., \& Levant, Y (2008). Cost modeling in logistics using time-driven ABC: Experiences from a wholesaler. International Journal of Physical Distribution \& Logistics Management, 38(3), 172191. doi: https://doi.org/10.1108/09600030810866977. 
Custeio Baseado em Atividade e Tempo (TDABC) Aplicado em Lavanderia: Estudo de Caso

Mariélly Warmeling Laucsen Martins, Clarice Flor Marçal, Rodney Wernke, Evandro Bonetti, Letícia

Sandrin

Fachini, G. J., Spessatto, G., \& Scarpin, J. E. (2008, Novembro). Utilização do timedriven activity-based costing como métrica do custo de processamento de pedidos de vendas. Anais do Congresso Brasileiro de Custos, Curitiba, PR, Brasil,15.

Fontoura, F. B. B. da (2013). Gestão de custos: uma visão integradora e prática dos métodos de custeio. São Paulo: Atlas.

Gervais, M., Levant, Y., \& Ducrocq, C. (2010, Summer). Time-driven activity-based costing (TDABC): an initial appraisal through a longitudinal case study. Journal of Applied Management Accounting Research, 8(2), 1-20.

Gil, A. C. (1999). Métodos e técnicas de pesquisa social. São Paulo: Atlas.

Instituto Brasileiro de Geografia e Estatística - IBGE. Brasil em Síntese.Recuperado de:<http://brasilemsintese.ibge.gov.br/servicos.html>

Instituto Brasileiro de Geografia e Estatística - IBGE (2012). Pesquisa anual de serviços, 2010. Rio de Janeiro: IBGE.

Kaplan, R. S. (2014). Improving value with TDABC. Healthcare Financial Management, 68(6), 76-83.

Kaplan, R. S., \& Anderson, S. R. (2004, November). Time-Driven Activity-Based Costing. Harvard Business Review, p. 18, Recuperado de: https://hbr.org/2004/11/time-driven-activity-based-costing.

Kaplan, R. S., \& Anderson, S. R. (2007). Custeio baseado em atividade e tempo. Rio de Janeiro: Campus.

Kont, K.-R., \& Jantson, S. (2011). Activity-based Costing (ABC) and Time-driven Activity-based Costing (TDABC): applicable methods for university libraries? Evidence Based Library and Information Practice, 6(4), 107-119. doi: http://dx.doi.org/10.18438/B8GG8Z

Luiz, G., Gasparetto, V., Lunkes, R. J., \& Schnorrenberger, D. (2014). Utilização do Método da Unidade de Esforço de Produção (UEP): estudo em uma empresa de cosméticos. Revista ABCustos, 9(1), 48-65.

Lukka, K. (2010). The roles and effects of paradigms in accounting research. Management Accounting Research, 21(2), 110-115.

Marques, K. C. M., Camacho, R. R., \& Alcantara, C. C. V. de. (2015). Avaliação do rigor metodológico de estudos de caso em contabilidade gerencial publicados em periódicos no Brasil. Revista Contabilidade \& Finanças, 26(67), 27-42. 
Custeio Baseado em Atividade e Tempo (TDABC) Aplicado em Lavanderia: Estudo de Caso

Mariélly Warmeling Laucsen Martins, Clarice Flor Marçal, Rodney Wernke, Evandro Bonetti, Letícia

Sandrin

Pereira, S. I. M. (2015). Custeio por atividades (ABC) e unidade de esforço de produção (UEP): similaridades, diferenças e complementaridades (Dissertação de Mestrado). Universidade de São Paulo - USP, São Paulo, SP, Brasil.

Pernot, Y E., Roodhooft, F., \& Van Den Abbeele, A. (2007). Time-driven Activity-based Costing for inter-library services: a case study in a university. The Journal of Academic Librarianship, 33(5), 551-560.

Ratnatunga, J., Tseb, M. S. C., \& Balachandranc, K. R. (2012). Cost management in Sri Lanka: a case study on volume, activity and time as cost drivers. The International Journal of Accounting, 47(3), 281-301.

Richardson, R. J. (1999). Pesquisa social: métodos e técnicas. São Paulo: Atlas.

Roldão, T. (2013, agosto 14). Indicador positivo. Jornal Diário Catarinense, p. 19.

Santos, L. C. B. dos., Vasconcelos, F. N. P. de., Colares, A. C. V.,\&Moreira, M. A. (2014). Profissionais da contabilidade engajados no auxílio gerencial às micros e pequenas empresas brasileiras. Revista Brasileira de Contabilidade, 43(210),57-69.

Sarokolaei, M. A., Savizb, M., Moradlooc, M. F., \& Dahajd, N. S. (2013). Time Driven Activity Based Costing by using Fuzzy logics. 2nd International Conference on Leadership, Technology and Innovation Management. Procedia - Social and Behavioral Sciences, 75, 338-345. doi: Http://dx.doi.org/10.1016/j.sbspro.2013.04.039

Siguenza-Guzman, L. (2014). Time-driven Activity-based Costing systems for cataloguing processes: a case study. Liber Quarterly, 23(2), 160-186.

Slavov, T. N. (2013). Gestão estratégica de custos: uma contribuição para a construção de sua estrutura conceitual (Tese de Doutorado). Faculdade de Economia, Administração e Contabilidade da Universidade de São Paulo - FEA/USP, São Paulo, SP, Brasil.

Souza, A. A. de., Avelar, E. A., Boina, T. M., \& Lara, C. O. (2009, Julho). Análise dos estudos empíricos realizados sobre o time-driven ABC entre os anos de 2004 e 2008. Anais do Congresso USP de Controladoria e Contabilidade, São Paulo, SP, Brasil, 9.

Tseb, M. S. C., \& Gong, M. Z. (2009). Recognition of idle resources in Time-driven Activity-based Costing and Resource Consumption Accounting Models. Journal of Applied Management Accounting Research, 7(2), 41-54.

Varila, M.,Seppanem, M., \& Suomala, P. (2007). Detailed cost modelling: a case study in warehouse logistics. International Journal of Physical Distribution \& Logistics Management, 37(3), 184-200. 
Custeio Baseado em Atividade e Tempo (TDABC) Aplicado em Lavanderia: Estudo de Caso Mariélly Warmeling Laucsen Martins, Clarice Flor Marçal, Rodney Wernke, Evandro Bonetti, Letícia

Wernke, R., Junges, I., \& Luiz, M. R. A. (2015). Proposta de planilha de custos para laboratório de próteses dentárias: estudo de caso com aplicação do TDABC. IberoamericanJ ournal of Industrial Engineering, 7(13), 216-236.

Yin, R. K. (2010). Estudo de caso: planejamento e método. Porto Alegre: Bookman.

Data de Submissão: 17/06/2016

Data de Aceite: 13/04/2017 